\title{
Vulvovaginal candidiasis and current perspectives: new risk factors and laboratory diagnosis by using MALDI TOF for identifying species in primary infection and recurrence
}

\author{
Lívia Custódio Pereira ${ }^{1,3}$ - Amabel Fernandes Correia ${ }^{2}$ Zita Dinis Lopes da Silva ${ }^{3}$. Ceres Nunes de Resende ${ }^{1}$. \\ Fabiana Brandão ${ }^{3}$. Rosane Mansan Almeida ${ }^{3}$. Yanna Karla de Medeiros Nóbrega ${ }^{3}$ (i)
}

Received: 24 August 2020 / Accepted: 16 February 2021 / Published online: 13 March 2021

(C) The Author(s) 2021

\begin{abstract}
Vulvovaginal candidiasis (VVC), considered the second cause of genital infection among women, has pathogenic mechanisms still to be elucidated and unknown risk factors. Prevalence studies with laboratory diagnosis (at first diagnosis and recurrence) are uncommon, especially using MALDI TOF, used in this clinical, epidemiological, and laboratory study for evaluating candidiasis, and identifying unknown risk factors. To obtain clinical and epidemiological data, patients were questioned, and there was material collection. Samples collected were identified by using phenotypic and presumptive methods and confirmed by MALDI TOF. This study analyzed 278 patients, divided into symptomatic $(n=173)$ and asymptomatic $(n=105)$ groups. Regarding the main candidiasis symptoms (discharge, itching, and burning), only $50.3 \%$ of patients described these concomitant symptoms, showing a positive predictive value of $67.8 \%$. Regarding the risk factors investigated, there was a statistical correlation between candidiasis and dairy products, gut transit, contraceptive use, respiratory allergy, and panty liners, describing new risk factors related to intestinal and vaginal dysbiosis. After Candida species analysis and confirmation, the primary prevalence was $80.9 \%$ (Candida albicans), 15.2\% (non-albicans), $1 \%$ (Rhodotorula mucilaginosa), and 1.9\% (unidentified species). In recurrence, the prevalence was $66.7 \%$ (C. albicans) and $33.3 \%$ (non-albicans). The presence of symptoms has low positive predictive value for the diagnosis of candidiasis, even when considering the classic triad of symptoms. Laboratory identification of yeast species is essential for correct treatment, preventing the resistance to antifungals and the high recurrence. In addition, dairy products and bowel habits, both related to intestinal and vaginal dysbiosis, may be associated with VVC.
\end{abstract}

Keywords Candidiasis $\cdot$ Candida $\cdot$ Non-albicans $\cdot$ Recurrence $\cdot$ Vaginal vulvovaginitis $\cdot$ Vaginal dysbiosis $\cdot$ Intestinal dysbiosis

\section{Introduction}

Candida spp. vulvovaginitis, or vulvovaginal candidiasis (VVC), considered the second cause of genital infection among women in menacme [1], occurs by excessive

Yanna Karla de Medeiros Nóbrega

yannanobrega@gmail.com

1 Vulvar Pathology Clinic, Department of Gynecology, Brasilia University Hospital, University of Brasilia, Brasília, DF, Brazil

2 Medical Biology Management, Center of Parasitology and Mycology, Central Public Health Laboratory of the District Federal (LACEN-DF), Brasília, DF, Brazil

3 Microbiology and Immunology Clinical Laboratory, Department of Pharmacy, Faculty of Health Sciences, University of Brasilia, Darcy Ribeiro Campus, Brasília, DF 70900-910, Brazil multiplication, favored by predisposing factors of yeast, found in the vaginal microbiota of women in reproductive phase [2, 3].

Despite the high prevalence and a large number of risk factors (RFs) associated with infection, the pathogenic VVC mechanisms and recurrent vulvovaginal candidiasis (VVCR) have not yet been fully elucidated [4]. Candida yeasts migrate from the lower gastrointestinal tract to the adjacent vestibule and vagina, a route similar to that taken by vaginal Lactobacillus species [5-7]. The vaginal colonization, as well as yeast growth and germination, is enhanced by an estrogenrich environment, which occurs in menacme, combined oral contraceptive use, and hormone replacement therapy [6].

Besides the estrogen-rich environment, other risk factors contribute to VVC development, including genetic factors determining host susceptibility to infection, inflammatory response development, vaginal microbiota dysbiosis [3, 8], 
sexual activity, hygiene and clothing habits, diseases such as diabetes mellitus [4], and atopy [9-11].

Among VVCR risk factors listed, vaginal microbiota dysbiosis, defined as an imbalance in this microbiota, has been most recently described as directly associated with infection pathogenesis. Vaginal microbiota (VMB) plays an important role in preventing colonization by pathogens [12] and is affected by internal and external factors including hormonal changes (estrogen), menstruation, intestinal microbiota (near the rectum), intimate hygiene habits, sexual interaction (sharing with a partner), and contraceptive use (COC) [13].

In this context, studies have shown that Lactobacillus spp., in particular L. crispatus, L. jensenii, and L. gasseri, are predominant in healthy VMB [14], acting through different mechanisms, and they form an important defensive barrier against Candida spp. infection [15-20].

Concerning diagnosis, although the clinical presentationcharacterized by discharge, intense itching, and burning, accompanied or not by vulvitis with edema and fissure presence-is frequent in VVC, these symptoms are not specific and may be associated to other clinical conditions, such as desquamative vaginitis, cytolytic vaginitis, aerobic and anaerobic vaginitis, trichomoniasis, gonorrhea, and allergic symptoms [21].

Fresh examination is based on direct microscopy, although an important diagnosis means fungal infections [22] have low sensitivity, varying from 40 to $70 \%$, because the result is dependent on microscopist experience [6]. In recurrent cases, culture is recommended; however, despite allowing species identification, it has low sensitivity $[6,23]$.

More recently, with the molecular age advent, methods employing DNA for fungal identification have been used which are able to provide accurate and faster results, demonstrating $>90 \%$ sensitivity in identifying Candida species [24-27].

Another alternative is mass spectrometry, an analytical technique that consists of the atoms or molecule ionization, their separation according to their mass/charge ratio $(\mathrm{m} / \mathrm{z})$, and then their identification and quantification [28]. At present, one of the most widely used methods for biomolecule analysis is matrix-assisted laser desorption ionization (MALDI), followed by detection in a time-of-flight (TOF) analyzer, MALDI TOF [29, 30]. MALDI TOF has been recognized as a fast and reliable tool for accurately identifying yeasts (more than $90 \%$ species identification specificity) since the spectra generated at identification are unique signatures of each microorganism [31-33].

Using these more accurate diagnostic methods, it has been possible to evaluate species prevalence involved in VVC etiology. Although about 80 to $90 \%$ have Candida albicans isolated as the etiological agent [2,34-38], non-albicans (NA) species, such as Candida glabrata, Candida krusei, Candida parapsilosis, and Candida tropicalis, are no longer considered commensal species to be understood as etiological VVC agents [3, 4, 39] which may be responsible for up to $30 \%$ of VVCR episodes [21, 40].

The more accurate determination of Candida species is particularly important in VVCR cases, whose prevalence demonstrated by some authors ranged from 5 to $10 \%$ [41-43]. A recent systematic review about overall VVCR prevalence has estimated that approximately 138 million women are affected annually, which corresponds to 3871 cases per 100,000 women/year and about 372 million women will be affected by life-long VVCR [44]. In this context, it is noteworthy that the recurrence prevalence obtained by prospective studies with laboratory diagnosis is unclear since most studies calculate the rates based on epidemiological questionnaires applied to patients, whose symptoms are self-described and can be confused with similar symptoms from another vaginitis.

Thus, facing a problem affecting women from different ethnicities worldwide, every effort should be made to study VVC and VVCR. This infection is very prevalent, and causes great discomfort, especially in the recurrent form, limiting the quality of life, affecting mental health and sexual life [45]. In this sense, this study aimed to identify the etiologic agent of infections as accurately as possible, characterizing new RFs associated with infection and evaluating recurrence with laboratory diagnosis.

\section{Materials and methods}

\section{Study design and ethics}

The sample was composed of 278 women attending gynecology outpatient clinics (Brasília, DF, Brazil). Of these, 105 were asymptomatic and $173 \mathrm{had}$ at least one vulvovaginitis symptoms, such as discharge, itching, burning, initial dyspareunia, dysuria, or malodor.

This study was conducted according to ethical principles (Declaration of Helsinki). All subjects signed an informed consent agreement to participate in this study, which was previously approved by the Research Ethics Committee (Faculdade de Medicina, Universidade de Brasília (CEPFM-UNB), protocol number 1,572,449).

\section{Epidemiological, clinical, and laboratorial screening}

\section{Epidemiological and clinical}

At the consultation, the researcher conducted an interview with the patient and completed the symptom and risk factor questionnaire (Table 1), and during the physical examination realized by an experienced gynecologist, a vaginal discharge sample was collected, in a swab with Stuart transport medium, and was sent for laboratory identification. 
Table 1 Symptom and risk factor questionnaire

\begin{tabular}{ll}
\hline Questions & Response choices \\
\hline Demographics & Age \\
& Marital status \\
Presence at of least one & Yes \\
symptom & No \\
Number of similar episodes & $\leq 1$ \\
in the last 12 months & $\leq 3$ \\
& $\geq 4$ \\
Symptoms (answers Yes or & $\geq 4$ \\
No) & Discharge, itching, burning, dyspareunia, \\
Medication use in the last 30 & foul odor, dysuria \\
days & Antibiotic, antifungal, or other \\
& \\
Antecedents and habits & Number of pregnancies, number of births \\
Antecedent obstetric & and number of abortions \\
Contraceptive method & Combined hormone, other (IUD, \\
condom, other)
\end{tabular}

IUD intrauterine device, $N A$ not applied

\section{Laboratorial}

Isolation and conservation of fungal isolates Fungal isolates were obtained from cultures at $35^{\circ} \mathrm{C}$ within $24 \mathrm{~h}$ in Sabouraud dextrose agar tubes with chloramphenicol (SDAC) (HiMedia, Mumbai, India). All isolated samples were stored at $50 \%$ aqueous glycerol solution and frozen in $2.0-\mathrm{mL}$ centrifuge microtubes at $-20{ }^{\circ} \mathrm{C}$ for recovery and subsequent control, if necessary, and sent for identification.

\section{Phenotypic identification of Candida species}

For the phenotypic identification, the germ tube test (GTT) and the presumptive identification in a chromogenic medium (CM) were performed.
GTT was performed to differentiate Candida albicans (GTT positive) from non-albicans Candida species (GTT negative). Four pure fresh colonies subcultured on SDAC for $24 \mathrm{~h}$ at $35^{\circ} \mathrm{C}$ were inoculated into $0.5 \mathrm{~mL}$ of human serum in test tubes $(5 \mathrm{~mL})$ and incubated at $35^{\circ} \mathrm{C}$ for $3 \mathrm{~h}$. Results were observed by an optical microscope $(\times 400)$ using slide and coverslip preparations. Positive results were considered as isolates that presented elongated projections, called "Germ Tubes." A reference American Type Culture Collection (ATCC) strain of Candida albicans (ATCC 90028) was employed as a positive control.

CM was used for presumptive identification of Candida albicans, Candida tropicalis, and Candida krusei. These Candida species are differentiated from the production of differently colored colonies due to reaction with the chromogenic substrates present in the medium. From subcultures on SDAC for $24 \mathrm{~h}$ at $35^{\circ} \mathrm{C}$, pure and fresh colonies were obtained and inoculated at $\mathrm{CM}$ with handle and then incubated for 24 to 48h. Plate reading was performed by visual means, where colony presence of specific color for each Candida species was observed. As a positive control, reference strains of Candida albicans ATCC 90028, Candida tropicalis ATCC 28707, and Candida krusei ATCC 34135 were used.

\section{Identification using matrix-assisted laser desorption ionization time-of-flight}

Mass spectrometry technique (MALDI TOF) use, a chemotaxonomic method for phenotypic identification of fungi, allowed the confirmatory identification of Candida spp.

The methodological technique principle uses whole yeast cells, which have molecular biomarkers, such as peptides or ribosomal proteins that are detected in the spectra generated after equipment reading. These protein spectra are characteristic of each fungal species, and functioning as the fingerprint of each microbial species, which are then compared to spectra deposited in databases that allow identification by comparability $[46,47]$.

Spectra were analyzed using VITEK MS® Knowledge Base (BioMérieux, Marcy-l'Étoile, France) version 3.0. For comparing peaks formed with the characteristic patterns of the microorganism species, genus, or family were performed, resulting in its identification. Results were considered valid when the percentages of identification probability were equal to or higher than $90.0 \%$ [48].

\section{VVCR evaluation}

During the study period, forty-one symptomatic patients were evaluated for recurrence, with the researcher reapplying the symptom and risk factor questionnaire and collecting another sample for laboratory evaluation. 


\section{Statistical analysis}

Statistical tests were performed using GraphPad Prism version 8.00 software for Windows (La Jolla, CA, USA). To analyze VVC risk factors, Fisher's exact test was used to verify if there was a correlation between VVC and the presence of each risk factor surveyed. The data were considered statistically significant when $p \leq 0.0500$. In calculating positive predictive value (PPV), equation PPV $=(a /(a+b))$ was used, where $a$ refers to patients with positive laboratory results, here called true positives, and $\mathrm{b}$, false positive results.

\section{Results}

\section{Population characterization}

This study included 278 women divided into symptomatic (62.2\%) and asymptomatic (37.8\%) groups. Regarding the positivity of laboratory tests, $53.2 \%$ of symptomatic women had positivity of laboratory tests; asymptomatic patients, $12.4 \%$ (Table 2).

The mean age was $37.4 \pm 7.7$ years, and the prevalent age group was 26 and 40 years old (59.6\% of the total), followed by the age group of 41 to 55 years. The age group $>55$ years was the one that included the smallest number (Table 2). Regarding marital status, patients declared themselves married $(61.3 \%)$ or single $(38.7 \%)$. Data from questionnaires (clinical data and risk factors) and laboratory tests are presented in Table 2.

\section{Symptom prevalence evaluation and laboratory diagnosis}

Regarding clinical aspects evaluated in symptomatic patients, the most frequent symptoms were discharge, itching, and burning, and only $50.3 \%$ of the patients described these symptoms concomitantly (Fig. 1).

When associated with laboratory diagnosis, symptom presence had a positive predictive value (PPV) of less than $60 \%$ in individual analysis and in the presence of the three most frequent concomitant symptoms, PPV was $67.8 \%$ (Table 3).

\section{Risk factor evaluation and laboratory diagnosis}

To analyze vulvovaginal candidiasis, risk factors were compared between symptomatic patients showing positive laboratory diagnosis (VVC group) and asymptomatic patients, with negative tests (control group) (Fig. 2).

Regarding the number of partners (last 12 months) and the time with the current partner, most patients in both groups had between one and two partners, and most women in both groups were with the same partner for more than one partner. No comparative analysis was performed for these two variables.

Patients in both groups were questioned about milk and dairy product consumption, which was categorized into less than one portion per day, or one or more portions per day. Comparative data analysis found that in the VVC group, ingestion was significantly higher when compared to the control group $(p<0.0001)$ (Fig. 2a).

Table 2 Group evaluation characterized by age, marital status and symptom presense

\begin{tabular}{|c|c|c|c|c|c|}
\hline Category & Symptomatic & & Asymptomatic & & Total \\
\hline \multirow[t]{2}{*}{$n$} & $173(62.2 \%)$ & & $105(37.8 \%)$ & & 278 \\
\hline & $\begin{array}{l}\text { Negative laboratory } \\
\text { diagnosis }\end{array}$ & $\begin{array}{l}\text { Positive laboratory } \\
\text { diagnosis }\end{array}$ & $\begin{array}{l}\text { Negative laboratory } \\
\text { diagnosis }\end{array}$ & $\begin{array}{l}\text { Positive laboratory } \\
\text { diagnosis }\end{array}$ & \\
\hline$n$ groups & $81(46.8 \%)$ & $92(53.2 \%)$ & $92(87.6 \%)$ & $13(12.4 \%)$ & \\
\hline \multicolumn{6}{|l|}{ Age } \\
\hline Media \pm SD & $38.5 \pm 5.0$ & $38.5 \pm 4.2$ & $38.5 \pm 11.3$ & $40 \pm 0.0$ & $37.4 \pm 7.7$ \\
\hline \multicolumn{6}{|l|}{ Age category } \\
\hline $19-25$ & $13(16.0 \%)$ & $8(8.7 \%)$ & $8(8.7 \%)$ & 0 & $29(10.3 \%)$ \\
\hline $26-40$ & $46(57.0 \%)$ & $61(66.3 \%)$ & $51(55.5 \%)$ & $7(53.8 \%)$ & $168(59.6 \%)$ \\
\hline $41-55$ & $17(21.0 \%)$ & $19(20.7 \%)$ & $27(29.3 \%)$ & $5(38.4 \%)$ & $69(24.5 \%)$ \\
\hline$>55$ & $5(6.0 \%)$ & $4(4.3 \%)$ & $6(6.5 \%)$ & $1(7.8 \%)$ & $16(5.6 \%)$ \\
\hline \multicolumn{6}{|l|}{ Marital status } \\
\hline Married & $46(56.8 \%)$ & $54(58.7 \%)$ & $62(67.4 \%)$ & $8(61.5 \%)$ & $170(61.1 \%)$ \\
\hline Single & $35(43.2 \%)$ & $38(41.3 \%)$ & $30(32.6 \%)$ & $5(38.5 \%)$ & $108(38.9 \%)$ \\
\hline Total & & & & & 278 \\
\hline
\end{tabular}

$S D$ standard deviation 
Fig. 1 Venn diagram in symptomatic patients

Patients were also asked about their bowel habits, and chronic constipation or diarrhea was considered as altered with gut transit. In the VVC group, there was a significantly higher frequency of intestinal transit alterations when compared to controls ( $p$ 0.0002) (Fig. 2b).

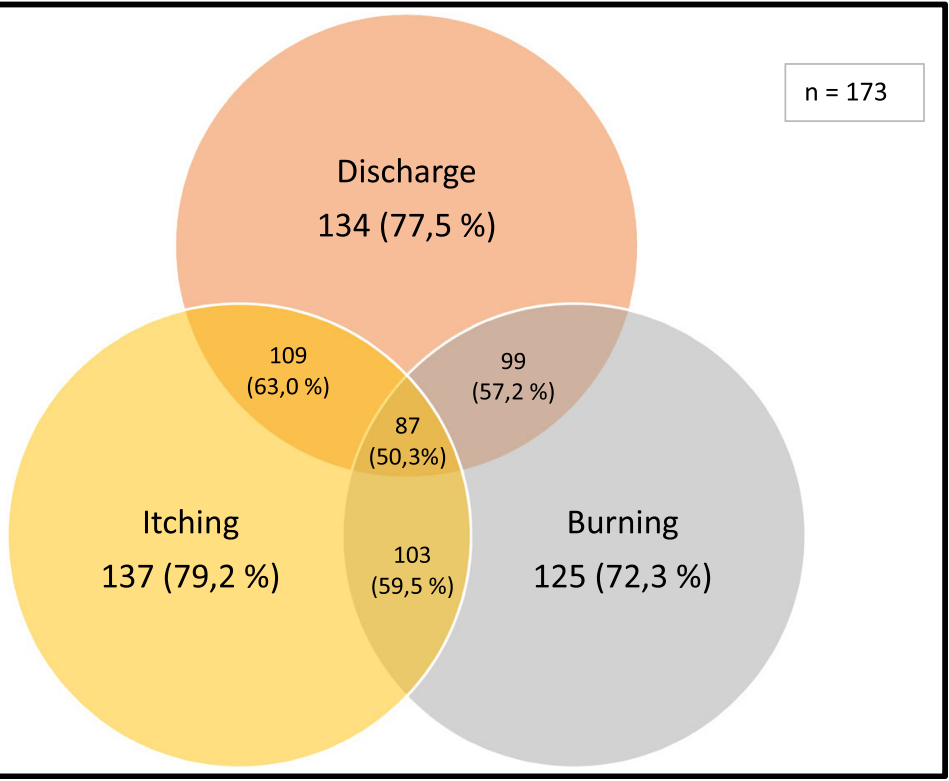

About hormonal contraceptives, the usual frequency was significantly higher among the VVC group when compared to the control group, $p<0.0001$ (Fig. 2c). Regarding condom use, there was no significant difference between the two groups, $p 0.1031$ (Fig. 2d).

Table 3 Symptom and laboratory diagnosis correlation

\begin{tabular}{|c|c|c|c|c|}
\hline \multicolumn{2}{|l|}{ Category } & \multicolumn{3}{|l|}{ Symptomatic } \\
\hline$n$ & & $173(61.3 \%)$ & & \\
\hline & Total & Negative laboratory diagnosis & Positive laboratory diagnosis & PPV \\
\hline$n$ groups & & $81(46.8 \%)$ & $92(53.2 \%)$ & \\
\hline \multicolumn{5}{|l|}{ Discharge } \\
\hline Yes & $39(22.5 \%)$ & $24(61.5 \%)$ & $15(38.5 \%)$ & $57.5 \%$ \\
\hline No & $134(77.5 \%)$ & $57(42.5 \%)$ & $77(57.5 \%)$ & \\
\hline \multicolumn{5}{|l|}{ Itching } \\
\hline Yes & $36(20.8 \%)$ & $26(72.2 \%)$ & $10(27.8 \%)$ & $59.8 \%$ \\
\hline No & $137(79.2 \%)$ & $55(40.1 \%)$ & $82(59.9 \%)$ & \\
\hline \multicolumn{5}{|l|}{ Burning } \\
\hline Yes & $48(27.7 \%)$ & $30(62.5 \%)$ & $18(37.5 \%)$ & $59.2 \%$ \\
\hline No & $125(72.3 \%)$ & $51(40.8 \%)$ & $74(59.2 \%)$ & \\
\hline Presence of discharge, itching, and burning & $87(50.3 \%)$ & $28(32.2 \%)$ & $59(67.8 \%)$ & $67.8 \%$ \\
\hline \multicolumn{5}{|l|}{ Dyspareunia } \\
\hline Yes & $83(48.0 \%)$ & $38(45.8 \%)$ & $45(54.2 \%)$ & $54.2 \%$ \\
\hline No & $87(50.3 \%)$ & $41(47.1 \%)$ & $46(52.9 \%)$ & \\
\hline NA & $3(1.7 \%)$ & $2(66.6)$ & $1(33.4 \%)$ & \\
\hline \multicolumn{5}{|l|}{ Bad odor } \\
\hline Yes & $155(89.6 \%)$ & $71(45.8 \%)$ & $84(54.2 \%)$ & $44.4 \%$ \\
\hline No & $18(10.4 \%)$ & $10(55.5 \%)$ & $8(44.5 \%)$ & \\
\hline \multicolumn{5}{|l|}{ Dysuria } \\
\hline Yes & $53(30.6 \%)$ & $19(37.8 \%)$ & $34(64.2 \%)$ & $64.2 \%$ \\
\hline No & $120(69.4 \%)$ & $62(51.7 \%)$ & $58(48.3 \%)$ & \\
\hline
\end{tabular}

$P P V$ positive predictive value 
a

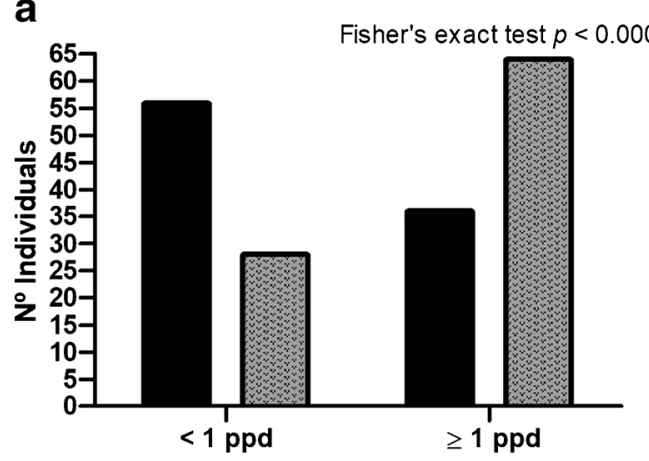

Milk and dairy products

C

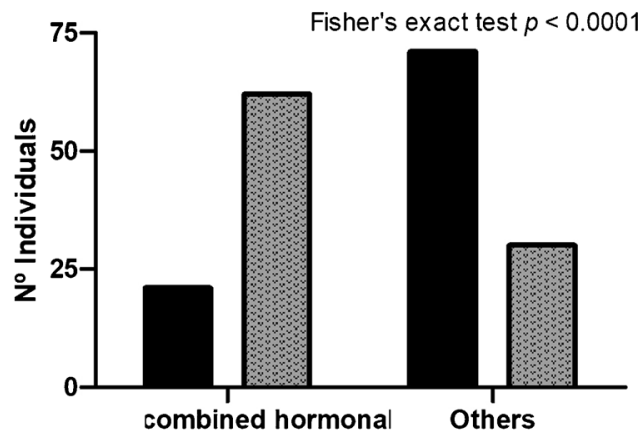

Contraceptive

e

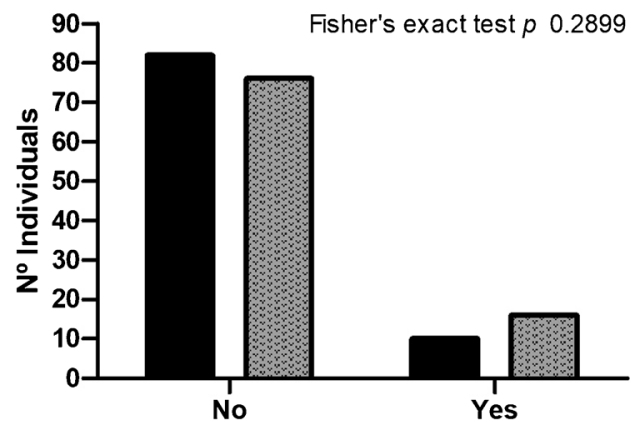

Cutaneous allergy

g

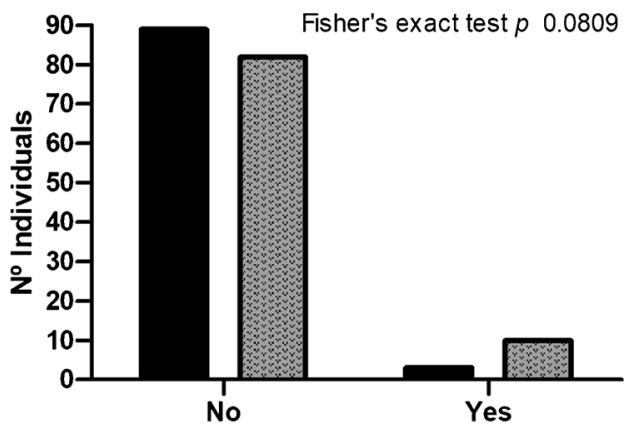

Vaginal douche
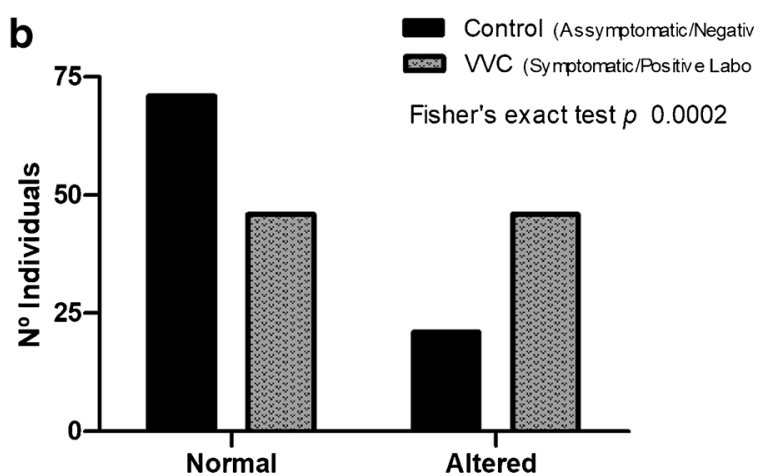

Gut transit

d

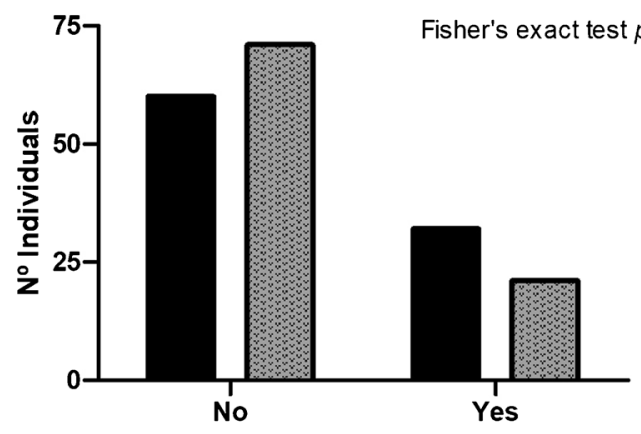

Condom

f

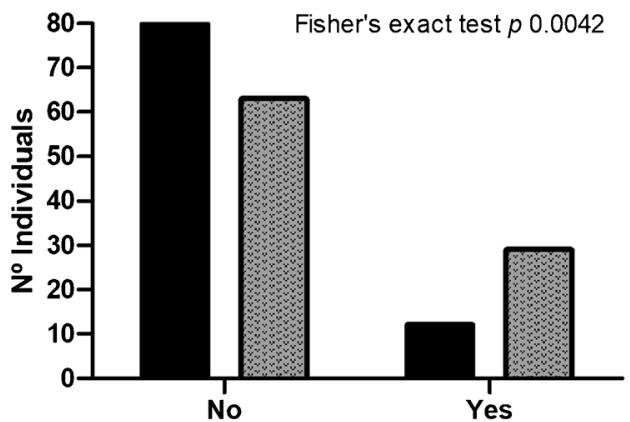

Respiratory allergy

h

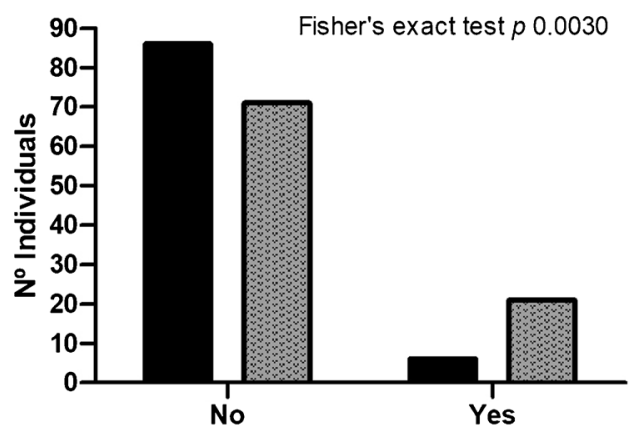

Panty Liners 
Fig. 2 a-h Analyzed risk factors of vulvovaginal candidiasis. Symptomatic patients showing positive laboratory diagnosis (VVC group) were compared with asymptomatic patients, with negative laboratory tests (control group)

Respiratory or skin allergies were also raised in both groups, and in relation to skin allergy, there was no significant difference between the two groups ( $p$ 0.2899) (Fig. 2e). However, in the case of respiratory allergy, the occurrence was significantly higher in the VVC group, with $p<0.0042$ (Fig. 2f).

Regarding personal habits, there was no significant difference between groups concerning vaginal douche use ( $p=$ 0.2899) (Fig. 2g); however, among VVC groups, the frequency of daily panty liner use was significantly higher, $p<0.0042$ (Fig. 2g).

\section{Candida species prevalence identified by laboratory diagnostic techniques}

Species identification was performed by phenotypic means of the GTT and CM and was confirmed by MALDI TOF. Using MALDI TOF analyses, among 173 patients in the VVC group, $92(53.2 \%)$ samples were positive for yeast, and among the 105 control group, $13(12.4 \%)$ samples were positive (Table 4)

GTT, which is positive only for $C$. albicans species, was positive in 75 samples among the VVC group, while the chromogenic medium identified 79 C. albicans-positive samples (Table 4), suggesting that GTT failed to identify 4 C. albicans samples identified by CM and confirmed by MALDI TOF, or these strains have mutated and do not produce germ tube.
For the analyses performed in CM, which distinguishes Candida species, except for the failure to identify another species of yeast, all results were in agreement to MALDI TOF. MALDI TOF confirmed the diagnosis of 78 C. albicans, and identified a species among the VVC group unidentified by either GTT or CM, confirmed as Rhodotorula mucilaginosa.

The comparison between the results obtained by MALDI TOF (gold standard) and those obtained by GTT and CM made it possible to calculate the specificity and sensitivity of these laboratory methods compared to the gold standard (Table 5), when used in symptomatic and asymptomatic patients, giving a precise idea of the evaluation and limitations of these methods, which can be used when it is impossible to use MALDI TOF.

Sample analysis by MALDI TOF allowed the species identification from eight different yeasts, with a predominance of C. albicans (80.9\%) followed by C. glabrata (6.6\%), C. parapsilosis, C. krusei, C. tropicalis, and C. zeylanoide (Fig. $3)$. One Candida species was not identified and is described in this study as Candida spp. (1.9\%) and another yeast, Rhodotorula mucilaginosa (1.0\%), was identified. Therefore, the prevalence of non-albicans species $(15.2 \%)$ from positive samples was identified (Fig. 3).

\section{Recurrence evaluation}

Among the 173 patients who were symptomatic during the first collection, 41 returned with symptoms and a second sample was collected (follow-up). Of these, 21 (51.2\%) had positive MALDI TOF samples in the first collection $(76.2 \% \mathrm{C}$. albicans, $23.8 \%$ other species). In the second collection, 6 $(28.6 \%)$ of these 21 patients remained with positive laboratory

Table 4 Laboratory diagnosis by phenotypic methods and MALDI TOF

\begin{tabular}{|c|c|c|c|c|c|c|}
\hline \multicolumn{7}{|c|}{ Laboratory diagnosis } \\
\hline \multicolumn{3}{|l|}{ Symptomatic } & \multicolumn{3}{|l|}{ Asymptomatic } & \multirow{3}{*}{$\begin{array}{l}\text { Total } \\
278\end{array}$} \\
\hline \multicolumn{3}{|l|}{$173(62.2 \%)$} & \multicolumn{3}{|l|}{$105(37.8 \%)$} & \\
\hline GTT & $\mathrm{CM}$ & MALDI TOF & GTT & $\mathrm{CM}$ & MALDI TOF & \\
\hline $\begin{array}{l}75 \text { positives } \\
(43.4 \%) \\
98 \text { negatives } \\
(56.6 \%) \\
74 \text { C. albicans } \\
(42.7 \%) \\
1 \text { Candida spp. } \\
(0.6 \%)\end{array}$ & $\begin{array}{l}90 \text { positives } \\
(52 \%) \\
82 \text { negatives } \\
(47.4 \%) \\
79 \text { C. albicans } \\
(48.4 \%) \\
12 \text { non-albicans } \\
(7.4 \%)\end{array}$ & $\begin{array}{l}92 \text { positives } \\
(53.2 \%) \\
81 \text { negatives } \\
(48.8 \%) \\
78 \text { C. albicans } \\
(45 \%) \\
13 \text { non-albicans } \\
(7.5 \%) \\
1 \text { other species } \\
(0.6 \%)\end{array}$ & $\begin{array}{l}6 \text { positives } \\
(5.7 \%) \\
99 \text { negatives } \\
(94.3 \%) \\
6 \text { C. albicans } \\
(5.7 \%)\end{array}$ & $\begin{array}{l}13 \text { positives } \\
(12.4 \%) \\
96 \text { negatives } \\
(87.6 \%) \\
7 \text { C. albicans } \\
(6.7 \%) \\
6 \text { non-albicans } \\
(5.7 \%)\end{array}$ & $\begin{array}{l}13 \text { positives }(12.4 \%) \\
96 \text { negatives } \\
(87.6 \%) \\
7 \text { C. albicans } \\
(6.7 \%) \\
6 \text { non-albicans } \\
(5.7 \%)\end{array}$ & \\
\hline
\end{tabular}

GTT (germ tube test) is positive only for C. albicans, $C M$ (chromogenic medium) differentiates yeast species from genus Candida 
Table 5 Sensitivity and specificity of the methodologies employed in comparison to the MALDI TOF gold standard

Methodologies employed

\begin{tabular}{|c|c|c|c|c|c|c|}
\hline & \multicolumn{3}{|l|}{ GTT } & \multicolumn{3}{|l|}{$\mathrm{CM}$} \\
\hline & Symptomatic & Asymptomatic & Media & Symptomatic & Asymptomatic & Media \\
\hline Sensitivity & $84 \%$ & $72 \%$ & $78 \%$ & $97 \%$ & $93 \%$ & $95 \%$ \\
\hline Specificity & $82 \%$ & $97 \%$ & $90 \%$ & $98 \%$ & $99 \%$ & $99 \%$ \\
\hline
\end{tabular}

GTT germ tube test, $C M$ chromogenic medium

diagnosis, showing $4(66.7 \%)$ identified as C. albicans samples and two (33.3\%) identified as non-albicans (Fig. 4).

\section{Discussion}

The diagnosis of VVC is very often based on the presence of characteristic symptoms. These diagnoses are used not only in clinical practice but also in most epidemiological studies on the prevalence of the disease. However, some studies indicate that, due to the low specificity, diagnosis based only on the presence of symptoms is very imprecise [6, 49] and, in the case of a self-reported diagnosis, it can be wrong by up to $90 \%$ $[33,50]$.

Despite this data, in practice, the treatment of VVC in most cases is based on clinical diagnosis, and there are few studies that assess the predictive value of symptoms compared to laboratory diagnosis. In the present study, the laboratory diagnosis confirmed by MALDI TOF, a highly sensitive method, was compared to the symptoms reported by patients, and, only about half of the symptomatic patients had positive laboratory results. Even when the classic triad of symptoms was reported, the PPV of symptoms to diagnosis VVC was also low confirming that similar symptoms appear in vulvovaginitis to other etiologies, as well as in cytolytic vaginitis, aerobic vaginitis, trichomoniasis, vulvar dermatosis, and atrophic vaginitis [49, 51-57], which reinforces the need for laboratory diagnosis.

With respect to VVC-related risk factors, those frequently described in previous studies, such as oral contraceptive use, condoms, vaginal douche, and daily panty liners $[4,6,58]$, were evaluated and, as well, those mentioned less frequently (respiratory and skin allergies) [10]. In addition to these risk factors already described, the study presented two new factors still little explored, namely the frequency of milk and dairy products, and changes in bowel habits. These factors were studied based on the current understanding that vaginal dysbiosis, defined as vaginal microbiota imbalance, may be directly implicated in the infection pathogenesis.
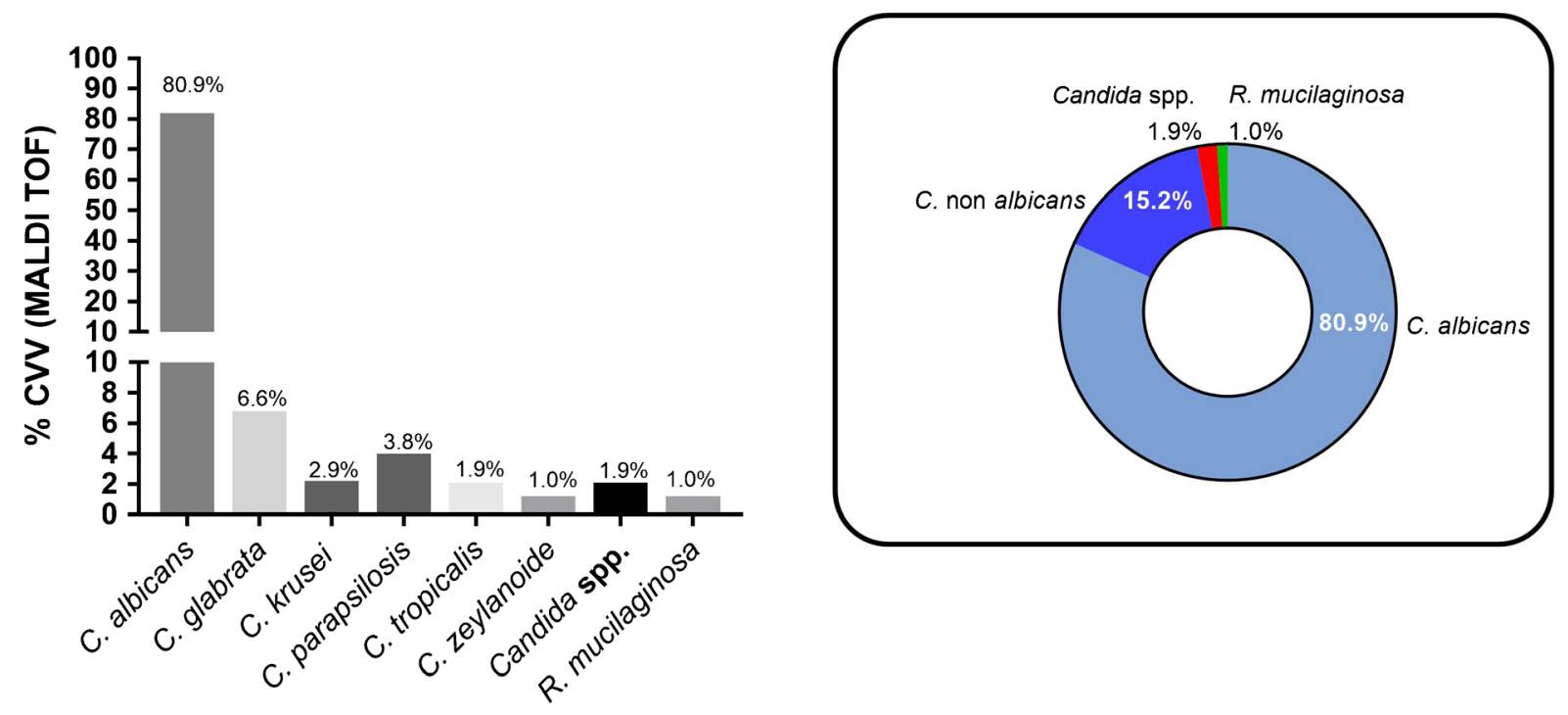

Fig. 3 Identification of yeasts species by MALDI TOF 


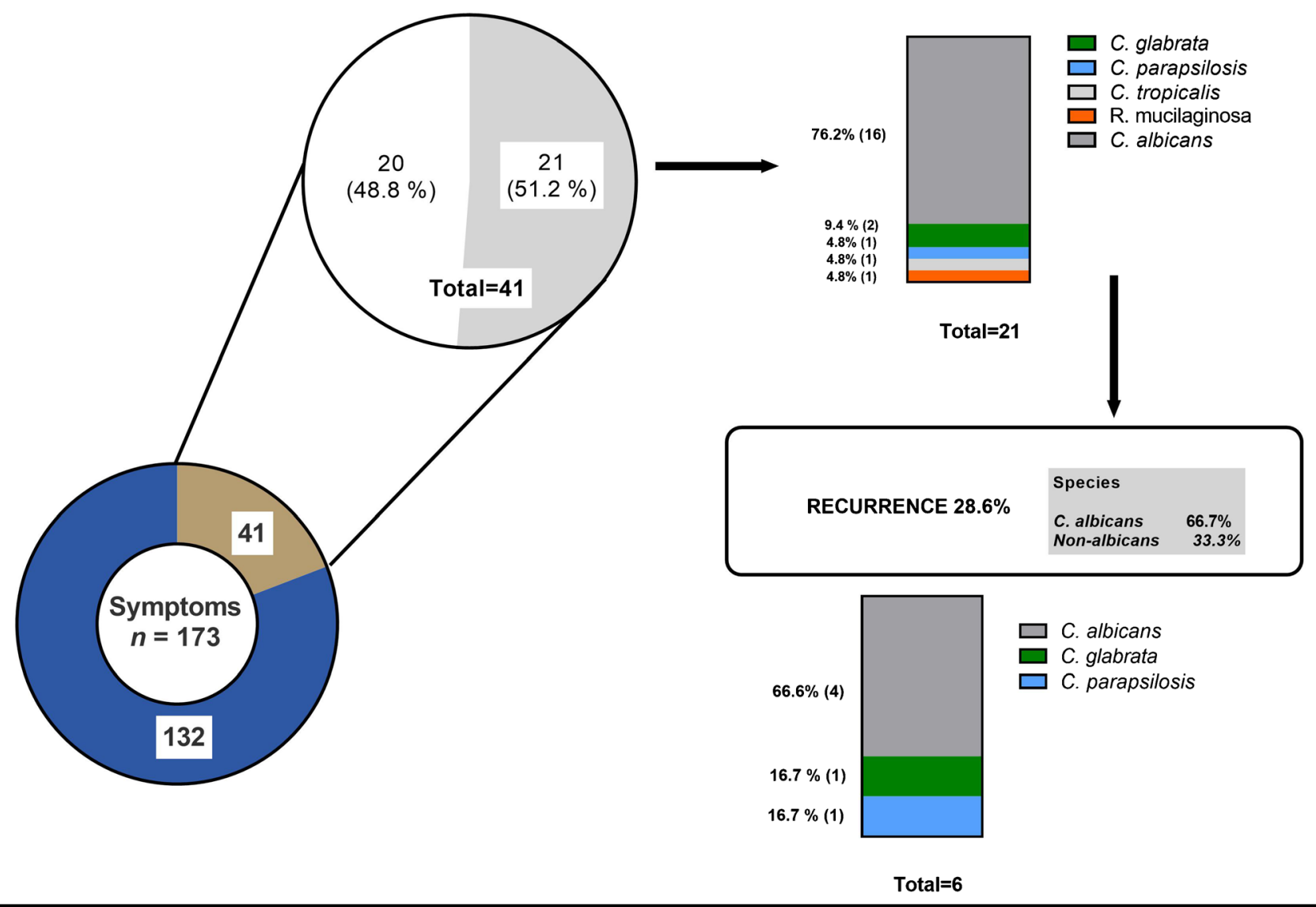

Fig. 4 Laboratory assessment of RVVC in symptomatic patients

The laboratory method for identifying Candida spp. was MALDI TOF, with more than $90 \%$ specificity $[32,33,59]$. This method requires expensive equipment, not always accessible for diagnosis. The realization of classic laboratory methodologies such as GTT and CM, although currently uncommon, allowed an assessment of the sensitivity and specificity of each of them concerning the gold standard (MALDI TOF). This result makes it possible to carry out laboratory diagnostics in laboratories that do not have the MALDI TOF methodology available to use, which would justify the use of GTT and CM methodologies in the study, and would possibility the use of these in similar studies, with knowledge of the expected sensitivity and specificity in symptomatic and asymptomatic patients.

Another factor to be highlighted is that the $\mathrm{CM}$ in addition to permit the division into two distinct groups, the albicans species of the non-albicans, enables the identification of more than one species of Candida spp., when present in the same biological sample, which suggests the existence of VVC by more than one species, and does not represent contamination of the analyzed biological sample (data are not shown).

For many authors, C. albicans is considered a VMB and IMB commensal [51, 53,60], and the onset of symptoms is attributed to a dysbiosis that results in excessive Candida growth $[6,11]$. However, data on asymptomatic colonization are quite limited. A study in Australia using CM for culture found a prevalence of Candida spp. $21 \%$ [61], while in the present study, the prevalence was $12.4 \%$.

This species is also the etiological agent of most cases of VVC [2, 33, 35-38, 62, 63]. Other authors pinpoint other species, such as Candida glabrata, Candida krusei, Candida parapsilosis, and Candida tropicalis as emerging [3, 4, 38]. In this study, the prevalence of Candida albicans (80.9\%) and non-albicans $(18.1 \%)$ was similar to previous studies. An unrelated yeast, Rhodotorula mucilaginosa, was also isolated. This species, considered non-virulent and saprophytic, was found in the genital region of $13.5 \%$ of asymptomatic people [64]. However, new studies show that it can be opportunistic $[65,66]$. Another study identified this yeast in $10 \%$ of vaginal samples from pregnant women, $3.8 \%$ of these symptomatic [67]. Although the prevalence of this species was very low here, it confirms the existence of VVC caused by this species.

VVC recurrence data based on prospective studies, including laboratory analyses of samples from the same patient collected at different times, are uncommon. Most studies assess recurrence based on questionnaires, such as a study that includes data from several countries, which indicated a VVCR 
of $9 \%$ [44]. Another survey found a VVCR of $34 \%$ among women who answered a questionnaire [50]. In this study, the examination of the same patient who remained symptomatic, performed at different times, revealed a recurrence rate of $28.6 \%$, and $33.3 \%$ for non-albicans species.

Some RFs that already described for VVC $[4,6,10,68]$ were evaluated. Confirming previous studies, the use of COC was greater when compared to controls. The use of a vaginal douche and condom did not differ between groups, perhaps due to the low prevalence of these habits.

The daily use of panty liners has been considered a risk factor for VVC [69, 70], but two reviews have not confirmed this association $[71,72]$. In contrast, in this study, the use of panty liners was higher in the VVC group ( $p$ 0.0003). However, it cannot be affirmed that using panty liners is a risk factor, since women with VVC may be more likely to use them due to the presence of abundant secretion.

Some studies have linked the co-existence of VVC to respiratory allergies (RA), although they do not explain a possible mechanism $[9,73,74]$. The VVC group reported RA more frequently in the present study ( $p$ 0.0042). The immune response in individuals with RA is mediated by Th17, which increases IL-10, an anti-inflammatory cytokine that transfers the immune response (IR) to a Th2 pole [75]. Some components of the cell wall of glucan-rich fungi negatively modulate IR, displacing it to Th2, favoring the persistence of fungi, and reducing microbicidal effects on it [76]. Thus, an individual with RA has more IL-10, and this cytokine-mediated IR is ineffective in eliminating fungi like Candida spp., suggesting that this atopic individual would be more susceptible to VVC.

The study also presented two new RFs, frequency of ingestion of milk and dairy products, and changes in bowel habits. These factors were studied based on the current understanding that vaginal dysbiosis may be implicated in infection. VMB plays an important role in preventing colonization by pathogens and acts in maintaining women's reproductive and gynecological health [12]. In addition, it is known that there is a correlation between IMB and VMB, including the terminal portion of the intestine as a source of colonization of the vagina by the migration of Lactobacillus spp. and yeasts $[6,51,77]$.

There is a peak of lactase expression in the small intestine villi at birth, and this expression decreases with age, decreasing the absorption of lactose, which in contact with the IMB is fermented, producing $\mathrm{H}_{2}, \mathrm{CO}_{2}$ and methane $\left(\mathrm{CH}_{4}\right)$, and shortchain fatty acids, which alter intestinal function [51]. Considering the most prevalent age group in the study, this deficiency in lactose absorption may result in an imbalance of the IMB, favoring the growth of yeasts, with interference in the $\mathrm{VMB}$, given the correlation between the microbiome of the two sites.

Patients were asked about their bowel habits and classified by Rome IV consensus [71, 72]. In the VVC group, there was a higher frequency of changes in intestinal transit ( $p$ 0.0002).
Studies show that, in individuals with intestinal constipation, bacteria of the genera Bifidobacterium and Lactobacillus decrease $[48,77-79]$. Thus, the decrease of these species in the intestine, especially Lactobacillus spp., which have a recognized role in maintaining vaginal health, possibly reduces their presence in the vagina, since the VMB is fed-back by the IMB.

Our study makes new contributions to the diagnosis and clinic of VVC. Laboratory diagnosis of VVC is essential for treatment based on the identification of the Candida species, since non-albicans species are present in many of these infections and some of them have intrinsic or acquired resistance to azoles, and this therapy would be ineffective against these pathogens. In addition, there is a need for this diagnosis to rule out the presence of another non-Candida vaginitis, which share the same classic symptoms of VVC (discharge, itching, and burning), the most frequently cited in the clinic, and which in our study proved that they did present a low PPV in the diagnosis of VVC in these patients.

The existence of new risk factors associated with CVV, such as milk and dairy products and bowel habits, in addition to the use of panty liners and the presence of respiratory allergies, is relevant to the diagnosis of CVV, and may be related to the presence of dysbiosis of vaginal and/or intestinal. And the inclusion of these risk factors can add information to the clinical anamnesis, especially in patients who have the recurrent form of candidiasis (VVCR).

\section{Strengths and limitations of the study}

Limitations in our study include the lack of a screening test, such as the Nugent criterion that could be performed using Gram stain, which would allow us to diagnose the presence of non-Candida vaginitis, in the absence and/or presence of CVV, making it possible to prove the existence of a single or mixed infection. Regarding the analysis of risk factors, the presence of a few patients using a vaginal shower and condom limited the analysis of these factors associated with CVV in the study.

The major strength of our study was to show the importance of laboratory diagnosis for the correct identification of $\mathrm{CVV}$, and to compare it with the prevalence of the literature, which is almost always based on self-diagnosis in questionnaires answered by patients who describe the main symptoms of CVV. The study identified that the PPV based on the presence of symptoms is low when confirmed by the laboratory diagnosis, emphasizing the importance of the laboratory diagnosis for the clinical diagnosis. In addition, the description of new risk factors may contribute to the physician's clinical conduct in conducting CVV treatment, namely the frequency of milk and dairy products, and changes in bowel habits, which may contribute to the understanding of vaginal dysbiosis. 


\section{Conclusion}

The presence of symptoms has low PPV for the diagnosis of candidiasis, even when considering the classic triad of symptoms. Laboratory identification of yeast species is essential for correct treatment, preventing the generation of resistance to antifungals, and the high prevalence of recurrent candidiasis as found in this study. In addition, new RFs, intake of dairy products, and bowel habits, both related to intestinal and vaginal dysbiosis, may be associated with VVC.

Author contribution Lívia Custódio Pereira recruited all patients, conducted all interviews, and wrote the manuscript; Amabel Fernandes Correia performed laboratory tests; Zita Dinis Lopes da Silva identified and sent for processing all the cultured samples; Ceres Nunes de Resende collected patient samples; Fabiana Brandão performed statistical analyses and reviewed and inserted all references; Rosane Mansan Almeida processed all results; and Yanna Karla de Medeiros Nóbrega designed the study and wrote the manuscript. All authors read and approved the final manuscript.

Funding The authors thank Coordenação de Aperfeiçoamento de Pessoal de Nivel Superior (CAPES) for its financial support, and the Graduate Program in Medical Sciences, School of Medicine, University of Brasilia, Brasília, DF, Brazil. This study was supported in part by grants from Central Public Health Laboratory of the District Federal (LACEN-DF), Medical Biology Management, Center of Parasitology and Mycology, Brasília, DF, Brazil.

\section{Declarations}

Ethical approval This study was previously approved by the Research Ethics Committee (Faculdade de Medicina, Universidade de Brasília (CEP-FM-UNB), protocol number 1,572,449) and is in accordance with the 1964 Helsinki Declaration.

Conflict of interest The authors declare no competing interests.

Open Access This article is licensed under a Creative Commons Attribution 4.0 International License, which permits use, sharing, adaptation, distribution and reproduction in any medium or format, as long as you give appropriate credit to the original author(s) and the source, provide a link to the Creative Commons licence, and indicate if changes were made. The images or other third party material in this article are included in the article's Creative Commons licence, unless indicated otherwise in a credit line to the material. If material is not included in the article's Creative Commons licence and your intended use is not permitted by statutory regulation or exceeds the permitted use, you will need to obtain permission directly from the copyright holder. To view a copy of this licence, visit http://creativecommons.org/licenses/by/4.0/.

\section{References}

1. Unemo M, Ballard R, Ison C, Lewis D, Ndowa F (2013) Laboratory diagnosis of sexually transmitted infections, including human immunodeficiency virus.

2. Sobel JD (1997) Vaginitis. N Engl J Med 337:1896-1903
3. Brandolt TM, Klafke GB, Gonçalves CV, Bitencourt LR, de Martinez AMB, Mendes JF, Meireles MCA, Xavier MO (2017) Prevalence of Candida spp. in cervical-vaginal samples and the in vitro susceptibility of isolates. Braz J Microbiol 48:145-150

4. Gonçalves B, Ferreira C, Alves CT, Henriques M, Azeredo J, Silva S (2016) Vulvovaginal candidiasis: epidemiology, microbiology and risk factors. Crit Rev Microbiol 42:905-927

5. van de Wijgert JHHM, Borgdorff H, Verhelst R, Crucitti T, Francis S, Verstraelen H, Jespers V (2014) The vaginal microbiota: what have we learned after a decade of molecular characterization? PLoS One 9:e105998

6. Sobel JD (2016) Recurrent vulvovaginal candidiasis. Am J Obstet Gynecol 214:15-21

7. Castro A, González M, Tarín JJ, Cano A (2015) Papel de los probióticos en Obstetricia y Ginecología. Nutr Hosp 31:26-29

8. Böcher S, Helmig RB, Arpi M, Bjerrum L (2018) [Diagnosis of vaginal discharge]. Ugeskr. Laeger 180

9. Moraes PS (1998) Recurrent vaginal candidiasis and allergic rhinitis: a common association. Ann Allergy Asthma Immunol 81:165169

10. Neves NA, Carvalho LP, De Oliveira MAM, Giraldo PC, Bacellar O, Cruz AA, Carvalho EM (2005) Association between atopy and recurrent vaginal candidiasis. Clin Exp Immunol 142:167-171

11. Donders GGG, Grinceviciene S, Bellen G, Jaeger M, ten Oever J, Netea MG (2018) Is non-response to fluconazole maintenance therapy for recurrent Candida vaginitis related to sensitization to atopic reactions? Am J Reprod Immunol 79:e12811

12. Martin DH, Zozaya M, Lillis R, Miller J, Ferris MJ (2012) The microbiota of the human genitourinary tract: trying to see the forest through the trees. Trans Am Clin Climatol Assoc 123:242-256

13. Reid G (2018) Has knowledge of the vaginal microbiome altered approaches to health and disease? F1000Res 7:460

14. Ravel J, Gajer P, Abdo Z et al (2011) Vaginal microbiome of reproductive-age women. Proc Natl Acad Sci U S A 108(Suppl 1):4680-4687

15. Kang C-H, Han SH, Kim Y, Paek N-S, So J-S (2018) In vitro probiotic properties of Lactobacillus salivarius MG242 isolated from human vagina. Probiotics Antimicrob Proteins 10:343-349

16. Chew SY, Cheah YK, Seow HF, Sandai D, Than LTL (2015) Probiotic Lactobacillus rhamnosus GR-1 and Lactobacillus reuteri RC-14 exhibit strong antifungal effects against vulvovaginal candidiasis-causing Candida glabrata isolates. J Appl Microbiol 118:1180-1190

17. Pendharkar S, Brandsborg E, Hammarström L, Marcotte H, Larsson P-G (2015) Vaginal colonisation by probiotic lactobacilli and clinical outcome in women conventionally treated for bacterial vaginosis and yeast infection. BMC Infect Dis 15:255

18. Jørgensen MR, Kragelund C, Jensen PØ, Keller MK, Twetman S (2017) Probiotic Lactobacillus reuteri has antifungal effects on oral Candida species in vitro. J Oral Microbiol 9:1274582

19. Malik S, Petrova MI, Imholz NCE et al (2016) High mannosespecific lectin Msl mediates key interactions of the vaginal Lactobacillus plantarum isolate CMPG5300. Sci Rep 6:37339

20. Faten K, Hamida K, Soumya EA, Saad ISK, Hasna M, Hassan L, Moktar H (2016) Lactobacillus plantarum: effect of a protective biofilm on the surface of olives during storage. Braz J Microbiol 47:202-209

21. Anderson MR, Klink K, Cohrssen A (2004) Evaluation of vaginal complaints. JAMA 291:1368-1379

22. Willinger B, Haase G (2013) State-of-the-art procedures and quality management in diagnostic medical mycology. Curr Fungal Infect Rep 7:260-272

23. Lease ED, Alexander BD (2011) Fungal diagnostics in pneumonia. Semin Respir Crit Care Med 32:663-672

24. Nguyen MH, Wissel MC, Shields RK et al (2012) Performance of Candida real-time polymerase chain reaction, $\beta$-d-glucan assay, 
and blood cultures in the diagnosis of invasive candidiasis. Clin Infect Dis 54:1240-1248

25. Tellapragada C, Eshwara VK, Johar R, Shaw T, Malik N, Bhat PV, Kamath A, Mukhopadhyay C (2014) Antifungal susceptibility patterns, in vitro production of virulence factors, and evaluation of diagnostic modalities for the speciation of pathogenic Candida from blood stream infections and vulvovaginal candidiasis. J Pathog 2014:142864

26. Avni T, Leibovici L, Paul M (2011) PCR diagnosis of invasive candidiasis: systematic review and meta-analysis. J Clin Microbiol 49:665-670

27. Ahmad S, Khan Z, Asadzadeh M, Theyyathel A, Chandy R (2012) Performance comparison of phenotypic and molecular methods for detection and differentiation of Candida albicans and Candida dubliniensis. BMC Infect Dis 12:230

28. Goulart VAM, de Sinalização Celular e Nanobiotecnologia L, Resende RR (2013) MALDI-TOF: uma ferramenta revolucionária para as análises clínicas e pesquisa do câncer. Nanocell News. https://doi.org/10.15729/nanocellnews.2013.11.21.001

29. Tanaka K (2003) The origin of macromolecule ionization by laser irradiation (Nobel lecture). Angew Chem Int Ed Eng 42:3860-3870

30. Wieser A, Schneider L, Jung J, Schubert S (2012) MALDI-TOF MS in microbiological diagnostics - identification of microorganisms and beyond (mini review). Appl Microbiol Biotechnol 93: 965-974

31. Croxatto A, Prod'hom G, Greub G (2012) Applications of MALDITOF mass spectrometry in clinical diagnostic microbiology. FEMS Microbiol Rev 36:380-407

32. Marklein G, Josten M, Klanke U et al (2009) Matrix-assisted laser desorption ionization-time of flight mass spectrometry for fast and reliable identification of clinical yeast isolates. J Clin Microbiol 47: 2912-2917

33. Alizadeh M, Kolecka A, Boekhout T, Zarrinfar H, Ghanbari Nahzag MA, Badiee P, Rezaei-Matehkolaei A, Fata A, Dolatabadi S, Najafzadeh MJ (2017) Identification of Candida species isolated from vulvovaginitis using matrix assisted laser desorption ionization-time of flight mass spectrometry. Curr Med Mycol 3:21-25

34. Sobel JD (2007) Vulvovaginal candidosis. Lancet 369:1961-1971

35. Gygax SE, Vermitsky J-P, Chadwick SG, Self MJ, Zimmerman JA, Mordechai E, Adelson ME, Trama JP (2008) Antifungal resistance of Candida glabrata vaginal isolates and development of a quantitative reverse transcription-PCR-based azole susceptibility assay. Antimicrob Agents Chemother 52:3424-3426

36. Spinillo A, Bernuzzi AM, Cevini C, Gulminetti R, Luzi S, De Santolo A (1997) The relationship of bacterial vaginosis, Candida and Trichomonas infection to symptomatic vaginitis in postmenopausal women attending a vaginitis clinic. Maturitas 27:253-260

37. Fan SR, Liu XP, Li JW (2008) Clinical characteristics of vulvovaginal candidiasis and antifungal susceptibilities of Candida species isolates among patients in southern China from 2003 to 2006. J Obstet Gynaecol Res 34:561-566

38. Ahmad A, Khan AU (2009) Prevalence of Candida species and potential risk factors for vulvovaginal candidiasis in Aligarh, India. Eur J Obstet Gynecol Reprod Biol 144:68-71

39. Nakamura-Vasconcelos SS, Fiorini A, Zanni PD, BonfimMendonça P d S, Godoy JR, Almeida-Apolonio AA, Consolaro MEL, Svidzinski TIE (2017) Emergence of Candida glabrata in vulvovaginal candidiasis should be attributed to selective pressure or virulence ability? Arch Gynecol Obstet 296:519-526

40. Kennedy MA, Sobel JD (2010) Vulvovaginal candidiasis caused by non-albicans Candida species: new insights. Curr Infect Dis Rep 12:465-470

41. Ilkit M, Guzel AB (2011) The epidemiology, pathogenesis, and diagnosis of vulvovaginal candidosis: a mycological perspective. Crit Rev Microbiol 37:250-261
42. Amouri I, Sellami H, Borji N et al (2011) Epidemiological survey of vulvovaginal candidosis in Sfax, Tunisia. Mycoses 54:e499 e505

43. Liu W, Zhang $X$, Liu Z, Luo X (2011) Impact of $p H$ on the antifungal susceptibility of vaginal Candida albicans. Int J Gynaecol Obstet 114:278-280

44. Denning DW, Kneale M, Sobel JD, Rautemaa-Richardson R (2018) Global burden of recurrent vulvovaginal candidiasis: a systematic review. Lancet Infect Dis 18:e339-e347

45. Blostein F, Levin-Sparenberg E, Wagner J, Foxman B (2017) Recurrent vulvovaginal candidiasis. Ann Epidemiol 27:575582.e3

46. Neppelenbroek KH, Seó RS, Urban VM, Silva S, Dovigo LN, Jorge JH, Campanha NH (2014) Identification of Candida species in the clinical laboratory: a review of conventional, commercial, and molecular techniques. Oral Dis 20:329-344

47. McCarty TP, Pappas PG (2016) Invasive candidiasis. Infect Dis Clin N Am 30:103-124

48. Kim Y, Park KG, Lee K, Park Y-J (2015) Direct identification of urinary tract pathogens from urine samples using the Vitek MS system based on matrix-assisted laser desorption ionization-time of flight mass spectrometry. Ann Lab Med 35:416-422

49. Donders GGG, Vereecken A, Bosmans E, Dekeersmaecker A, Salembier G, Spitz B (2005) Aerobic vaginitis: abnormal vaginal flora entity that is distinct from bacterial vaginosis. Int Congr Ser 1279:118-129

50. Yano J, Sobel JD, Nyirjesy P, Sobel R, Williams VL, Yu Q, Noverr MC, Fidel PL Jr (2019) Current patient perspectives of vulvovaginal candidiasis: incidence, symptoms, management and post-treatment outcomes. BMC Womens Health 19:48

51. Nikou S-A, Kichik N, Brown R, Ponde NO, Ho J, Naglik JR, Richardson JP (2019) Candida albicans interactions with mucosal surfaces during health and disease. Pathogens. https://doi.org/10. 3390/pathogens 8020053

52. Odds FC (1988) Chronic mucocutaneous candidosis. Candida and Candidosis.

53. Bernardis FD, De Bernardis F, Arancia S, Sandini S, Graziani S, Norelli $S$ (2015) Studies of immune responses in Candida vaginitis. Pathogens 4:697-707

54. Neal CM, Kus LH, Eckert LO, Peipert JF (2019) Non-candidal vaginitis: a comprehensive approach to diagnosis \& management. Am J Obstet Gynecol

55. Paavonen J, Brunham RC (2018) Bacterial vaginosis and desquamative inflammatory vaginitis. N Engl J Med 379:22462254

56. Hu Z, Zhou W, Mu L, Kuang L, Su M, Jiang Y (2015) Identification of cytolytic vaginosis versus vulvovaginal candidiasis. J Low Genit Tract Dis 19:152-155

57. Yang S, Zhang Y, Liu Y, Wang J, Chen S, Li S (2017) Clinical significance and characteristic clinical differences of cytolytic vaginosis in recurrent vulvovaginitis. Gynecol Obstet Investig 82:137143

58. Chatzivasileiou P, Vyzantiadis T-A (2019) Vaginal yeast colonisation: from a potential harmless condition to clinical implications and management approaches-a literature review. Mycoses 62: $638-650$

59. Shah HN, Gharbia SE (2017) MALDI-TOF and tandem MS for clinical microbiology. John Wiley \& Sons

60. Odds FC (1988) Candida and candidosis. Elsevier Science Health Science Division

61. Pirotta MV, Garland SM (2006) Genital Candida species detected in samples from women in Melbourne, Australia, before and after treatment with antibiotics. J Clin Microbiol 44:3213-3217

62. Hetticarachchi N, Ashbee HR, Wilson JD (2010) Prevalence and management of non-albicans vaginal candidiasis. Sexually transmitted 
63. Vermitsky J-P, Self MJ, Chadwick SG, Trama JP, Adelson ME, Mordechai E, Gygax SE (2008) Survey of vaginal-flora Candida species isolates from women of different age groups by use of species-specific PCR detection. J Clin Microbiol 46:1501-1503

64. Bentubo HDL, Mantovani A, Yamashita JT, Gambale W, Fischman O (2015) Yeasts of the genital region of patients attending the dermatology service at Hospital São Paulo, Brazil. Rev Iberoam Micol 32:229-234

65. Wirth F, Goldani LZ (2012) Epidemiology of Rhodotorula: an emerging pathogen. Interdiscip Perspect Infect Dis 2012:465717

66. Bizerra FC, Ferreira RC, Colombo AL (2013) Molecular identification, antifungal susceptibility profile, and biofilm formation of clinical and environmental Rhodotorula species isolates. Antimicrob Agents Chemother

67. Mucci MJ, Cuestas ML, Cervetto MM, Landaburu MF, Mujica MT (2016) A prospective observational study of vulvovagintis in pregnant women in Argentina, with special reference to candidiasis. Mycoses 59:429-435

68. Farage M, Bramante M, Otaka Y, Sobel J (2007) Do panty liners promote vulvovaginal candidiasis or urinary tract infections? A review of the scientific evidence. Eur J Obstet Gynecol Reprod Biol 132:8-19

69. Miles MR (1977) Recurrent vaginal candidiasis. Importance of an intestinal reservoir. JAMA 238:1836-1837

70. Misselwitz B, Butter M, Verbeke K, Fox MR (2019) Update on lactose malabsorption and intolerance: pathogenesis, diagnosis and clinical management. Gut 68:2080-2091

71. Drossman DA (2016) Functional gastrointestinal disorders: history, pathophysiology, clinical features and Rome IV. Gastroenterology. https://doi.org/10.1053/j.gastro.2016.02.032
72. Mearin F, Lacy BE, Chang L, Chey WD, Lembo AJ, Simren M, Spiller R (2016) Bowel disorders. Gastroenterology. https://doi.org/ 10.1053 /j.gastro.2016.02.031

73. Surber C, Elsner P, Farage MA (2011) Topical applications and the mucosa. Karger Medical and Scientific Publishers

74. Guo F, Yang Y, Kang Y et al (2013) Invasive candidiasis in intensive care units in China: a multicentre prospective observational study. J Antimicrob Chemother 68:1660-1668

75. Rabin RL, Levinson AI (2008) The nexus between atopic disease and autoimmunity: a review of the epidemiological and mechanistic literature. Clin Exp Immunol 153:19-30

76. Nóbrega YK d M, Lozano VF, de Araújo TS, de Carvalho DD, Bocca AL (2010) The cell wall fraction from Fonsecaea pedrosoi stimulates production of different profiles of cytokines and nitric oxide by murine peritoneal cells in vitro. Mycopathologia 170:89 98

77. Parthasarathy G, Chen J, Chen X, Chia N, O'Connor HM, Wolf PG, Gaskins HR, Bharucha AE (2016) Relationship between microbiota of the colonic mucosa vs feces and symptoms, colonic transit, and methane production in female patients with chronic constipation. Gastroenterology 150:367-79.e1

78. Khalif I, Quigley E, Konovitch E, Maximova I (2005) Alterations in the colonic flora and intestinal permeability and evidence of immune activation in chronic constipation. Dig Liver Dis 37:838849

79. Chassard C, Dapoigny M, Scott KP et al (2012) Functional dysbiosis within the gut microbiota of patients with constipatedirritable bowel syndrome. Aliment Pharmacol Ther 35:828-838

Publisher's note Springer Nature remains neutral with regard to jurisdictional claims in published maps and institutional affiliations. 